\title{
The intralaminar thalamus - an expressway linking visual stimuli to circuits determining agency and action selection
}

\author{
Simon D. Fisher and John N. J. Reynolds* \\ Department of Anatomy, Brain Health Research Centre, School of Medical Sciences, University of Otago, Dunedin, Otago, New Zealand
}

Edited by:

Gavan McNally, University of New

South Wales, Australia

Reviewed by:

Bernard W. Balleine, University of

Sydney, Australia

David Tai Hsu, University of Michigan, USA

\section{*Correspondence:}

John N. J. Reynolds, Department of

Anatomy, Brain Health Research

Centre, School of Medical Sciences,

University of Otago, P.O. Box 913,

Dunedin 9054, Otago, New Zealand

e-mail:john.reynolds@otago.ac.nz

\begin{abstract}
Anatomical investigations have revealed connections between the intralaminar thalamic nuclei and areas such as the superior colliculus (SC) that receive short latency input from visual and auditory primary sensory areas. The intralaminar nuclei in turn project to the major input nucleus of the basal ganglia, the striatum, providing this nucleus with a source of subcortical excitatory input. Together with a converging input from the cerebral cortex, and a neuromodulatory dopaminergic input from the midbrain, the components previously found necessary for reinforcement learning in the basal ganglia are present. With this intralaminar sensory input, the basal ganglia are thought to play a primary role in determining what aspect of an organism's own behavior has caused salient environmental changes. Additionally, subcortical loops through thalamic and basal ganglia nuclei are proposed to play a critical role in action selection. In this mini review we will consider the anatomical and physiological evidence underlying the existence of these circuits. We will propose how the circuits interact to modulate basal ganglia output and solve common behavioral learning problems of agency determination and action selection.
\end{abstract}

Keywords: intralaminar, basal ganglia, learning, plasticity, sensory, action selection, agency

\section{INTRODUCTION}

An early view of the function of the intralaminar thalamus attached a nonspecific role to these nuclei in propagating reticular arousal signals (Steriade and Glenn, 1982; Groenewegen and Berendse, 1994). Recent anatomical and electrophysiological evidence has, however, painted the intralaminar nuclei in a much more behaviorally influential light. Through functional relationships with primary sensory areas and the basal ganglia, these nuclei have emerged as key contributors to circuits that underlie behavioral learning and selection processes.

The foundation for this new role emanated from early anatomical descriptions of ascending projections from the midbrain superior colliculus (SC) to the thalamic nuclei. A general division of the SC was proposed by Harting et al. (1973) between the superficial visual layers, which project to the thalamic visual centers described at that time, and the deep motor and arousal layers, which project to the non-visual thalamic intralaminar nuclei, and other subcortical motor and arousal areas. The intralaminar nuclei had in turn been found by Cowan and Powell (1956) to contribute a major projection to the striatum, the primary input nucleus of the basal ganglia.

A primary characteristic of the SC is its particular sensitivity to the appearance of salient stimuli, such as novel objects and loud or bright events (Wurtz and Albano, 1980; Sparks, 1986). It responds to such stimuli at very short latency, preceding any gaze shift intended to bring a newly appearing stimulus into view, and therefore is an ideal candidate to provide early sensory signals to subcortical processing units. The intralaminar nuclei have been recently shown to play a critical role in allowing this short-latency signal to gain access to circuits in the basal ganglia.
Recent studies have demonstrated that the appearance of a salient sensory event will lead to a temporal convergence in the striatum of a sensory signal via the intralaminar nuclei (Schulz et al., 2009) and a dopaminergic signal (Dommett et al., 2005). These inputs have been considered in previous work as necessary substrates for changes in synaptic efficacy at corticostriatal synapses, a cellular mechanism thought to underlie reinforcement learning in the basal ganglia (Reynolds and Wickens, 2002). One function that relies on reinforcement learning processes at shortlatency is the ability to determine agency-to discover causal relationships in our world between actions and their outcomes (Redgrave et al., 2011). Another function that exploits the convergence of short-latency signals, via proposed tecto-thalamo-striatal loops, is action selection (McHaffie et al., 2005).

In this mini review we will précis the anatomical and physiological evidence underlying the existence of subcortical loops between the SC, intralaminar and striatal circuits. We will propose how changes in synaptic efficacy via the short-latency convergence of critical learning signals can resolve the problems of agency determination and behavioral selection.

\section{ANATOMY}

The mammalian SC is a laminated midbrain structure consisting of three layers: the superficial, intermediate and the deep layers. These can be subdivided, based on cytoarchitecture, into a total of seven layers, alternating between cell-rich and fiber-rich layers (Kanaseki and Sprague, 1974). See May (2006) for an extensive cross-species review of the structure.

The superficial layers are primarily visual, receiving direct retinal input together with indirect visual information from areas 
of the visual cortex. These layers project to visual thalamic nuclei, including the lateral geniculate and pulvinar nuclei, and also down to the deep layers of the SC (Harting et al., 1992; May, 2006). The deep layers are multimodal and incorporate inputs from somatosensory and auditory sources, together with nonsensory modulatory inputs from cortical and subcortical areas (Gaese and Johnen, 2000). Anatomical studies in the rat reveal that the intermediate and deep SC layers send glutamatergic projections to both rostral and caudal intralaminar thalamic nuclei (Krout et al., 2001; Van der Werf et al., 2002). The caudal intralaminar nuclei comprise the center median and parafascicular $(\mathrm{CM} / \mathrm{Pf})$ nuclei in primates. In rodents, the lateral portion of the Pf is homologous to the primate CM (Groenewegen and Berendse, 1994; Van der Werf et al., 2002). The rostral intralaminar nuclei comprise the central medial, paracentral and central lateral nuclei. It is noteworthy that the thalamic nuclei targeted by the SC are those that provide the primary thalamic input to the basal ganglia (Takada et al., 1985; Féger et al., 1994).

The major target of the intralaminar nuclei, previously thought to be cortical, is the striatum (Parent et al., 1983; Smith and Parent, 1986; Sadikot et al., 1992). Via synapses onto striatal spiny neurons, the intralaminar nuclei modify the output from the striatum through either the direct or indirect pathway to the principal output nuclei of the basal ganglia: the substantia nigra pars reticulata $(\mathrm{SNr})$ and the internal globus pallidus (GPi), respectively. The basal ganglia outputs return direct projections to the caudal intralaminar CM/Pf complex from the GPi (Kuo and Carpenter, 1973; DeVito and Anderson, 1982; Parent et al., 1983; Sidibé et al., 1997) and the SNr (de las Heras et al., 1998; Sidibé et al., 2002; Tsumori et al., 2003). The basal ganglia output nuclei also project to the midbrain, including the SC (Redgrave et al., 1992), and indirectly via the thalamus to cortical and limbic regions from which basal ganglia input originated (Alexander et al., 1986; Parent and Hazrati, 1995; Smith et al., 2004). See Figure 1A for an abstracted high-level view of these structures and relations.

The subcortical structures reviewed here, including the SC (Stein and Gaither, 1981), thalamic nuclei (Butler, 1994), and basal ganglia (Reiner et al., 1998), are all evolutionarily ancient, and appear well-conserved in vertebrates. It has been proposed that these highly interconnected subcortical structures evolved to function together for survival in primitive organisms, prior to the emergence of the cortex in higher order vertebrates (McHaffie et al., 2005).

\section{REINFORCEMENT LEARNING}

One of the first functional roles ascribed to the SC was to direct eye gaze towards or away from salient events, owing to its sensitivity to novel objects and loud or bright stimuli (Wurtz and Albano, 1980; Sparks, 1986). SC neurons respond with shortlatency bursts of activity, tightly coupled to stimulus onset (Jay and Sparks, 1987). Putative spiny neurons in the dorsal striatum are also activated by salient visual stimuli, with a short-latency (100-150 ms) response that typically precedes the gaze shift time required (150-200 ms) to foveate to a salient visual event (Sparks, 1986; Hikosaka et al., 1989). This suggests that the responses of the

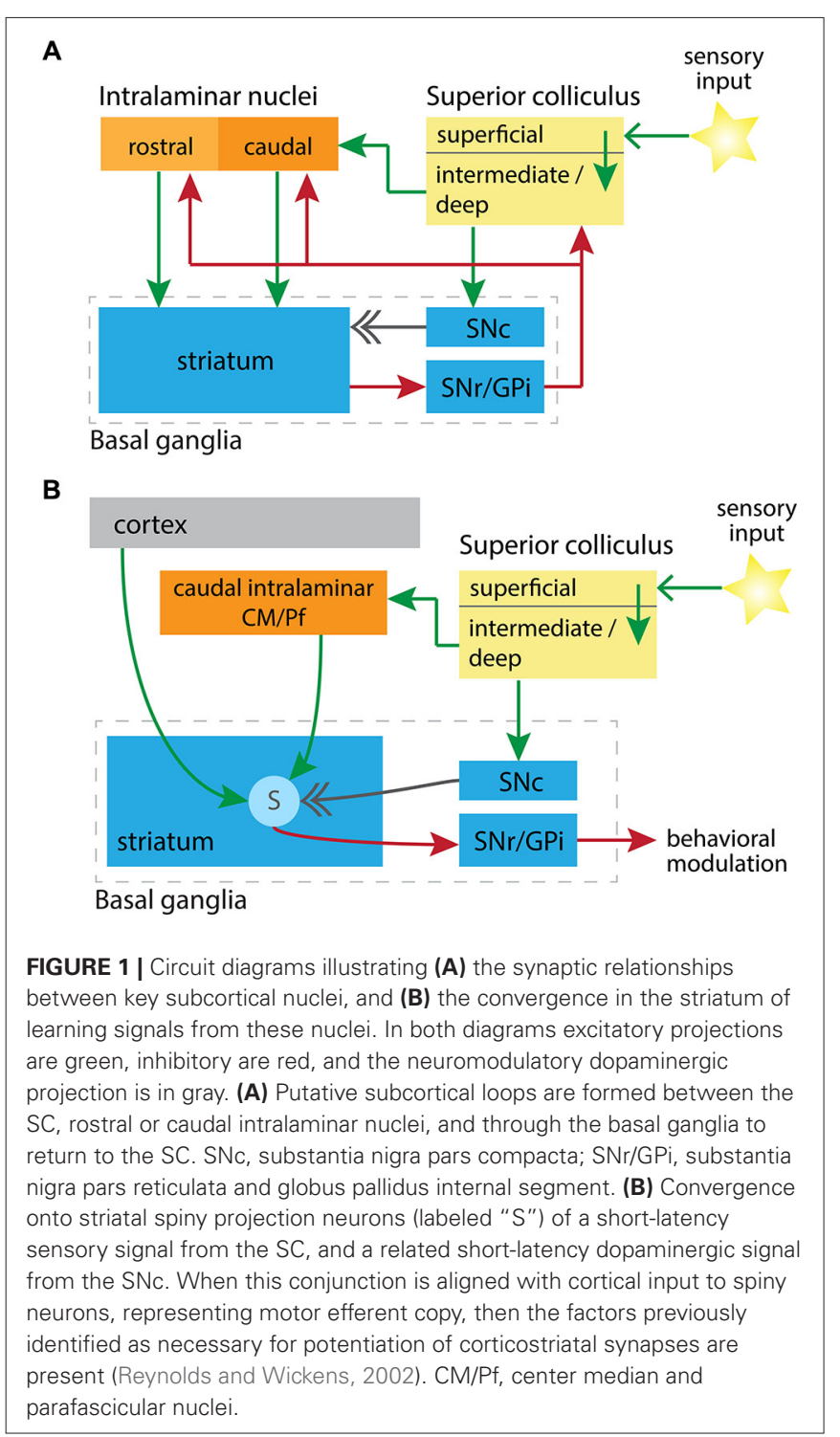

SC and dorsal striatal neurons are involved in early, pre-foveation, processing of important environmental events.

It was recently shown that the short-latency striatal response is mediated by a pathway from the SC to the striatum via the intralaminar nuclei (Figure 1B). Schulz et al. (2009) found that light flashes presented to a rat's eye drove dorsal striatal spiny neurons to their depolarized "up state" within a latency of approximately $115 \mathrm{~ms}$. This visually-evoked response was abolished with inhibitory GABA agonists injected into the $\mathrm{CM} / \mathrm{Pf}$ complex, revealing the reliance on the caudal intralaminar nuclei.

The SC has also been shown to be critical in transmitting short-latency visual information to dopamine neurons (Comoli et al., 2003), which are known for their phasic responses to unexpected, biologically-salient stimuli (Schultz et al., 1997). Notably, this activation causes phasic dopamine release in the striatum at approximately $110 \mathrm{~ms}$ following the event (Dommett 
et al., 2005), coinciding with the aforementioned dopamineindependent visually-evoked response in the spiny neurons. Moreover, there is evidence to suggest that both the striatal and dopaminergic responses to visual stimuli may be due to collaterals of the same SC projection (Coizet et al., 2007). Hence, there is a temporal convergence on striatal spiny neurons of excitatory thalamic input from the caudal intralaminar nuclei and dopamine from the SNc, informing the basal ganglia about the occurrence of salient events and providing neuromodulatory signals required for reinforcement learning (Wickens et al., 2003; Redgrave et al., 2008). The intralaminar nuclei also extensively innervate cholinergic interneurons in the striatum (Lapper and Bolam, 1992) and via these inputs mediate context-specific reinforcement learning mechanisms, specifically in dorsomedial striatum (Bradfield et al., 2013).

In addition to thalamic and dopamine projections, spiny neurons receive a vast input from almost all areas of the cerebral cortex, including collateral copies of ongoing motor commands from the motor cortices (Reiner et al., 2003). Changes in synaptic efficacy at these corticostriatal synapses are thought to underlie reinforcement learning, strengthening a motor representation by potentiating the efficacy of its corticostriatal projections. The cortical motor inputs, coupled with the convergence of sensory and dopamine signals described above, may present the necessary substrates to satisfy a three-factor learning rule required for longterm potentiation (LTP) at corticostriatal synapses (Reynolds and Wickens, 2002; Figure 1B). Pre-synaptic activity provided by the cortical inputs to be modified, in conjunction with thalamic activation from the intralaminar nuclei, may co-operate in some as yet unspecified manner to provide spiny neurons with the necessary signals and drive LTP by an appropriately timed phasic dopamine reinforcement signal. Phasic dopamine responses have also been shown to modulate corticostriatal plasticity in cholinergic interneurons (Suzuki et al., 2001; Reynolds and Wickens, 2004), however the association between this plasticity and learning has yet to be directly demonstrated in these interneurons, as it has with spiny neurons (Reynolds et al., 2001).

Thus far we have evidence of short-latency sensory and dopaminergic responses to salient visual stimuli that can simultaneously converge on spiny neurons via tecto-thalamo-striatal and tecto-nigro-striatal routes. Coupled with cortical motor signals, these components have the ability to modify corticostriatal weighting, a key control point in the cortico-basal ganglia loops. What could be the functional role of this system, which appears adept at responding quickly to salient sensory events, prior to the full processing that occurs post gaze orientation?

\section{AGENCY DETERMINATION}

A fundamental issue for an organism presented with an unexpected external event is to determine agency, i.e., if they were the agent causing the event, and which of their actions in particular was responsible. This is a problem in neural processing terms, as at any time during normal behavior a plethora of motor and sensory inputs are available, and only a small proportion are likely to relate to a recent action that caused a salient environmental event. This issue is critical in reinforcement learning in general, as an agent must be able to form actionoutcome associations, in order to perform the same action in similar future circumstances and achieve the same desirable outcome.

The short-latency sensory and dopamine inputs are well suited for an instrumental role in a basal ganglia system that determines agency (Redgrave et al., 2011). To determine agency, the three factors of reinforcement learning previously described, including a short-latency signal via the intralaminar nuclei, are critical. Hence, a behavior causing an appropriately timed salient environmental event might be represented in the striatum by an excitatory cortical input representing the motor copy, followed by a convergence of excitatory thalamic input from the SC and dopaminergic input from the SNc. By creating the conditions for potentiation at corticostriatal synapses (Reynolds and Wickens, 2002), it has been proposed that this arrangement of inputs conveys to the organism that it was the agent that caused the sensory event by emitting a particular action (Redgrave et al., 2011). If, conversely, the convergence of thalamic and dopaminergic input arrived in the striatum in the absence of a preceding motor copy input, then this might convey to the organism that it was not the agent of that external environmental event.

In agency computations, short-latency signals are required so that processing can be performed before the relevant motor and sensory signals become contaminated with signals from the response to the sensory event, such as a gaze shift. Additionally, the shorter the latency, the greater the minimization of noise contributed by non-causal cortical signals. Repetition of potentiated actions by trial and error might strengthen the associations between the causal components of behavior and the outcome by dopamine-dependent LTP, and weaken non-causal coincidental behavior patterns via LTD (Reynolds and Wickens, 2002; Wickens et al., 2003; Redgrave et al., 2011). In this way agency determination would be resolved.

\section{ACTION SELECTION}

The existence of segregated, parallel loops involving the basal ganglia has been proposed as an elegant solution to the problem of behavioral selection (Redgrave et al., 1999, 2011). Selection becomes necessary due to the inherent conflicts arising when the large array of distributed sensory, cognitive and affective systems in the brain require access to the limited motor plant by which to initiate a movement response. Through its segregated loop architecture, the basal ganglia nuclei are able to process multiple competing actions simultaneously, and return neural activity associated with the action with the most desired outcome back to the sites of originating inputs. By removing inhibition of particular representations via its output nuclei, the basal ganglia can thus initiate a selected action (Chevalier and Deniau, 1990; Mink, 1996).

In recent years an appreciation of the importance of subcortical loops in action selection has been emerging ( $\mathrm{McHaffie}$ et al., 2005; Redgrave et al., 2010). McHaffie et al. (2005) proposed the existence of at least three functionally segregated subcortical loop systems involving the SC and thalamic nuclei. Two of these loops are of particular relevance to this review (Figure 1A). 
First, the deep layer-rostral intralaminar thalamic loop, describing the connectivity from the deep SC layers, through the rostral intralaminar nuclei, and into the striatum. Second, the deep layercaudal intralaminar thalamic loop, passing instead through the caudal intralaminar nuclei. These loops are considered segregated due to the different thalamic routes and also the nature of their thalamo-striatal synaptic contacts. Each exhibits different terminal arborization (Deschênes et al., 1995; Parent and Parent, 2005), synaptic contact points (Sidibé and Smith, 1999; Raju et al., 2006), and neuronal targets (Sidibé and Smith, 1996; Smith et al., 2004) across the dorsal and ventral striatum. In these cases, loops are formed by projections back to the SC from both major output nuclei of the basal ganglia, the SNr (Hopkins and Niessen, 1976; Redgrave et al., 1992) and the GPi (Takada et al., 1994).

With respect to the processing of salient visual events via the $\mathrm{SC}$, there are at least two levels of selection operating. First, as the SC represents multiple stimuli in parallel, it must be able to selectively focus on a limited set of stimuli, and initiate a gaze shift towards a target. This selection case is likely handled via a non-intralaminar circuit from upper SC layers, through visual thalamic nuclei (e.g., lateral posterior nucleus), into the basal ganglia; and resulting in modulation of SC activity via disinhibition from the $\mathrm{SNr}$, together with excitatory input from gaze centers (Takada et al., 1985; Hikosaka et al., 2006). Second, once visual selection of salient stimuli has occurred, the organism can select an appropriate early behavioral responsesuch as orient and approach, or withdraw and defend-based on the primitive stimuli features initially available. For instance, the SC is activated by dark "looming" stimuli in a number of species (Westby et al., 1990; Billington et al., 2011; Liu et al., 2011) and SC stimulation can elicit orientation and approach, or freezing and withdrawal responses, depending on the stimulation site (Sahibzada et al., 1986). Taking the rodent SC as a model, it mediates approach and withdrawal responses in distinct lateral and medial regions, respectively (Dean et al., 1986, 1989; Comoli et al., 2012), which are likely to have differential thalamic projections and potentially align with segregated tecto-thalamo-striatal intralaminar loops (Chevalier and Deniau, 1984). At the output of the basal ganglia, the nigro-tectal pathway is organized topographically so that the ventromedial SNr projects exclusively to the medial SC, while the dorsolateral SNr projects exclusively to the lateral SC (Redgrave et al., 1992; Comoli et al., 2012). Hence, it is possible that early selection of approach or withdrawal behavioral responses may be performed based on short-latency sensory inputs from the SC via subcortical loops through the intralaminar nuclei. Complete details of the functional roles of the SC-intralaminar loops await full elucidation.

\section{COMPETITION BETWEEN CORTICAL AND SUBCORTICAL LOOPS}

Competition between subcortical and cortical loops has been proposed (McHaffie et al., 2005), a competition biased by the anatomy of subcortical looped structures (Figure 2). For instance, there is a markedly greater density of thalamostriatal synapses on spiny neurons of the direct pathway (Sidibé and Smith, 1996; Huerta-Ocampo et al., 2013), whereas corticostriatal synapses

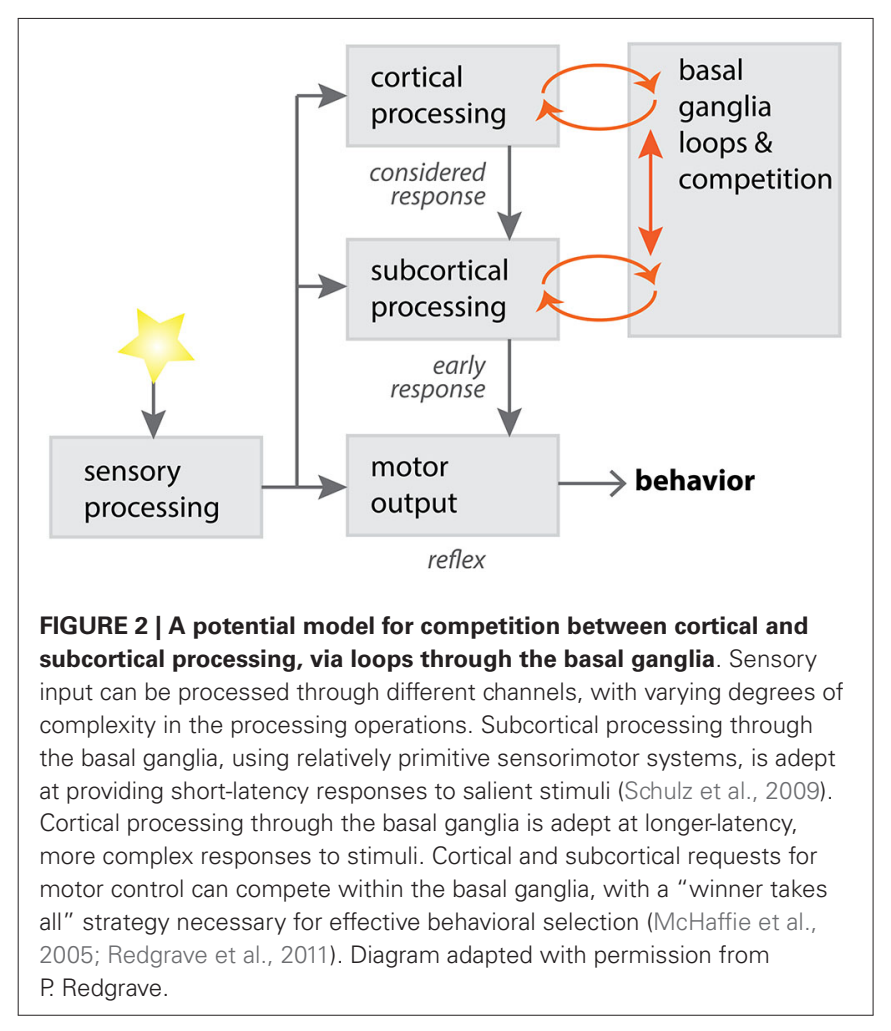

are more evenly distributed between direct and indirect pathway neurons. As the direct pathway is thought to be responsible for action initiation (Albin et al., 1989; Alexander and Crutcher, 1990; Kravitz et al., 2010), subcortical thalamic loops have the potential to rapidly direct motor resources in response to a salient stimulus.

The deep layer-caudal intralaminar thalamic loop may also gain an advantage in motor control over cortical loops via its preferential innervation of cholinergic interneurons in the striatum. An important aspect of a selection system is to be able to quickly switch attentional and motor resources to a new salient stimulus - for instance one that might require fight or flight. To do this, all ongoing motor programs need to be rapidly inhibited. Short-latency SC-intralaminar signals would be optimally placed to perform this role, due to the SC's access to salient sensory events, and the strong CM/Pf control of cholinergic striatal interneurons (Schulz et al., 2011).

In response to salient visual stimuli, tonically-active neurons in the striatum (putative cholinergic interneurons), exhibit a burst-pause sequence of activity (Morris et al., 2004; Goldberg and Reynolds, 2011; Schulz and Reynolds, 2013). This pattern requires an intact thalamic input from CM/Pf (Matsumoto et al., 2001), and can be induced by thalamic activation in an in vitro model (Ding et al., 2010). During the burst phase of the response, acetylcholine selectively enhances dendritic excitability of indirect pathway spiny neurons, with the net effect of biasing activation of indirect spiny neurons and increasing outflow from the indirect pathway (Shen et al., 2007). As the indirect pathway is generally thought to mediate motor suppression (Wichmann and DeLong, 1996), this circuit appears to be specialized for rapidly arresting 
ongoing motor activity in response to unexpected salient stimuli (Freeze et al., 2013).

In summary, the intralaminar nuclei, through their connections with the SC and basal ganglia, provide the striatum with short-latency access to salient stimuli important for behavioral learning and selection. Through connections with cholinergic interneurons, the caudal intralaminar pathway is able to exploit the basal ganglia motor control systems to suppress ongoing behavior and rapidly respond to a salient stimulus. The physiological advantages the subcortical loops have over cortical loops in the basal ganglia may explain how unpredicted sensory events, for example a bright light flash in the periphery, can rapidly command control of attentional and motor resources. This would appear to be evolutionarily advantageous to the survival of vertebrate organisms. Further, dysfunction of this circuit may be relevant to human psychiatric conditions characterized by hypervigilance, such as anxiety, or disordered agency, such as schizophrenia.

\section{ACKNOWLEDGMENTS}

The authors thank Prof P. Redgrave, University of Sheffield, for helpful discussions. During the preparation of this article, John N. J. Reynolds received support from a Rutherford Discovery Fellowship from the Royal Society of New Zealand.

\section{REFERENCES}

Albin, R. L., Young, A. B., and Penney, J. B. (1989). The functional anatomy of basal ganglia disorders. Trends Neurosci. 12, 366-375. doi: 10.1016/01662236(89)90074-x

Alexander, G. E., and Crutcher, M. D. (1990). Functional architecture of basal ganglia circuits: neural substrates of parallel processing. Trends Neurosci. 13, 266-271. doi: 10.1016/0166-2236(90)90107-1

Alexander, G. E., DeLong, M. R., and Strick, P. L. (1986). Parallel organization of functionally segregated circuits linking basal ganglia and cortex. Annu. Rev. Neurosci. 9, 357-381. doi: 10.1146/annurev.neuro.9.1.357

Billington, J., Wilkie, R. M., Field, D. T., and Wann, J. P. (2011). Neural processing of imminent collision in humans. Proc. Biol. Sci. 278, 1476-1481. doi: 10. 1098/rspb.2010.1895

Bradfield, L. A., Bertran-Gonzalez, J., Chieng, B., and Balleine, B. W. (2013). The thalamostriatal pathway and cholinergic control of goal-directed action: interlacing new with existing learning in the striatum. Neuron 79, 153-166. doi: 10.1016/j.neuron.2013.04.039

Butler, A. B. (1994). The evolution of the dorsal thalamus of jawed vertebrates, including mammals: cladistic analysis and a new hypothesis. Brain Res. Brain Res. Rev. 19, 29-65. doi: 10.1016/0165-0173(94)90003-5

Chevalier, G., and Deniau, J. M. (1984). Spatio-temporal organization of a branched tecto-spinal/tecto-diencephalic neuronal system. Neuroscience 12, 427-439. doi: 10.1016/0306-4522(84)90063-0

Chevalier, G., and Deniau, J. M. (1990). Disinhibition as a basic process in the expression of striatal functions. Trends Neurosci. 13, 277-280. doi: 10. 1016/0166-2236(90)90109-n

Coizet, V., Overton, P. G., and Redgrave, P. (2007). Collateralization of the tectonigral projection with other major output pathways of superior colliculus in the rat. J. Comp. Neurol. 500, 1034-1049. doi: 10.1002/cne.21202

Comoli, E., Coizet, V., Boyes, J., Bolam, J. P., Canteras, N. S., Quirk, R. H., et al. (2003). A direct projection from superior colliculus to substantia nigra for detecting salient visual events. Nat. Neurosci. 6, 974-980. doi: 10.1038/ nn1113

Comoli, E., Das Neves Favaro, P., Vautrelle, N., Leriche, M., Overton, P. G., and Redgrave, P. (2012). Segregated anatomical input to sub-regions of the rodent superior colliculus associated with approach and defense. Front. Neuroanat. 6:9. doi: 10.3389/fnana.2012.00009

Cowan, W. M., and Powell, T. P. (1956). A study of thalamo-striate relations in the monkey. Brain 79, 364-390. doi: 10.1093/brain/79.2.364 de las Heras, S., Mengual, E., and Giménez-Amaya, J. M. (1998). Overlapping territories between the thalamostriatal and nigrothalamic projections in cats. Neuroreport 9, 1913-1916. doi: 10.1097/00001756-199806010-00045

Dean, P., Redgrave, P., Sahibzada, N., and Tsuji, K. (1986). Head and body movements produced by electrical stimulation of superior colliculus in rats: effects of interruption of crossed tectoreticulospinal pathway. Neuroscience 19, 367-380. doi: 10.1016/0306-4522(86)90267-8

Dean, P., Redgrave, P., and Westby, G. W. (1989). Event or emergency? Two response systems in the mammalian superior colliculus. Trends Neurosci. 12, 137-147. doi: 10.1016/0166-2236(89)90052-0

Deschênes, M., Bourassa, J., and Parent, A. (1995). Two different types of thalamic fibers innervate the rat striatum. Brain Res. 701, 288-292. doi: 10.1016/00068993(95)01124-3

DeVito, J. L., and Anderson, M. E. (1982). An autoradiographic study of efferent connections of the globus pallidus in Macaca mulatta. Exp. Brain Res. 46, 107117. doi: $10.1007 / \mathrm{bf} 00238104$

Ding, J. B., Guzman, J. N., Peterson, J. D., Goldberg, J. A., and Surmeier, D. J. (2010). Thalamic gating of corticostriatal signaling by cholinergic interneurons. Neuron 67, 294-307. doi: 10.1016/j.neuron.2010.06.017

Dommett, E., Coizet, V., Blaha, C. D., Martindale, J., Lefebvre, V., Walton, N., et al. (2005). How visual stimuli activate dopaminergic neurons at short latency. Science 307, 1476-1479. doi: 10.1126/science.1107026

Féger, J., Bevan, M., and Crossman, A. R. (1994). The projections from the parafascicular thalamic nucleus to the subthalamic nucleus and the striatum arise from separate neuronal populations: a comparison with the corticostriatal and corticosubthalamic efferents in a retrograde fluorescent double-labelling study. Neuroscience 60, 125-132. doi: 10.1016/0306-4522(94)90208-9

Freeze, B. S., Kravitz, A. V., Hammack, N., Berke, J. D., and Kreitzer, A. C. (2013). Control of Basal Ganglia output by direct and indirect pathway projection neurons. J. Neurosci. 33, 18531-18539. doi: 10.1523/jneurosci.1278-13.2013

Gaese, B. H., and Johnen, A. (2000). Coding for auditory space in the superior colliculus of the rat. Eur. J. Neurosci. 12, 1739-1752. doi: 10.1046/j.1460-9568. 2000.00054.x

Goldberg, J. A., and Reynolds, J. N. J. (2011). Spontaneous firing and evoked pauses in the tonically active cholinergic interneurons of the striatum. Neuroscience 198, 27-43. doi: 10.1016/j.neuroscience.2011.08.067

Groenewegen, H. J., and Berendse, H. W. (1994). The specificity of the "nonspecific" midline and intralaminar thalamic nuclei. Trends Neurosci. 17, 52-57. doi: 10.1016/0166-2236(94)90074-4

Harting, J. K., Hall, W. C., Diamond, I. T., and Martin, G. F. (1973). Anterograde degeneration study of the superior colliculus in Tupaia glis: evidence for a subdivision between superficial and deep layers. J. Comp. Neurol. 148, 361-386. doi: 10.1002/cne.901480305

Harting, J. K., Updyke, B. V., and Van Lieshout, D. P. (1992). Corticotectal projections in the cat: anterograde transport studies of twenty-five cortical areas. J. Comp. Neurol. 324, 379-414. doi: 10.1002/cne.903240308

Hikosaka, O., Nakamura, K., and Nakahara, H. (2006). Basal ganglia orient eyes to reward. J. Neurophysiol. 95, 567-584. doi: 10.1152/jn.00458.2005

Hikosaka, O., Sakamoto, M., and Usui, S. (1989). Functional properties of monkey caudate neurons. II. Visual and auditory responses. J. Neurophysiol. 61, 799-813.

Hopkins, D. A., and Niessen, L. W. (1976). Substantia nigra projections to the reticular formation, superior colliculus and central gray in the rat, cat and monkey. Neurosci. Lett. 2, 253-259. doi: 10.1016/0304-3940(76)90156-7

Huerta-Ocampo, I., Mena-Segovia, J., and Bolam, J. P. (2013). Convergence of cortical and thalamic input to direct and indirect pathway medium spiny neurons in the striatum. Brain Struct. Funct. doi: 10.1007/s00429-013-0601-z. [Epub ahead of print].

Jay, M. F., and Sparks, D. L. (1987). Sensorimotor integration in the primate superior colliculus. I. Motor convergence. J. Neurophysiol. 57, 22-34.

Kanaseki, T., and Sprague, J. M. (1974). Anatomical organization of pretectal nuclei and tectal laminae in the cat. J. Comp. Neurol. 158, 319-337. doi: 10.1002/cne. 901580307

Kravitz, A. V., Freeze, B. S., Parker, P. R. L., Kay, K., Thwin, M. T., Deisseroth, K., et al. (2010). Regulation of parkinsonian motor behaviours by optogenetic control of basal ganglia circuitry. Nature 466, 622-626. doi: 10.1038/nature09159

Krout, K. E., Loewy, A. D., Westby, G. W., and Redgrave, P. (2001). Superior colliculus projections to midline and intralaminar thalamic nuclei of the rat. J. Comp. Neurol. 431, 198-216. doi: 10.1002/1096-9861(20010305)431:2<198::aidcne1065>3.0.co;2-8 
Kuo, J. S., and Carpenter, M. B. (1973). Organization of pallidothalamic projections in the rhesus monkey. J. Comp. Neurol. 151, 201-236. doi: 10.1002/cne. 901510302

Lapper, S. R., and Bolam, J. P. (1992). Input from the frontal cortex and the parafascicular nucleus to cholinergic interneurons in the dorsal striatum of the rat. Neuroscience 51, 533-545. doi: 10.1016/0306-4522(92)90293-b

Liu, Y.-J., Wang, Q., and Li, B. (2011). Neuronal responses to looming objects in the superior colliculus of the cat. Brain Behav. Evol. 77, 193-205. doi: 10. $1159 / 000327045$

Matsumoto, N., Minamimoto, T., Graybiel, A. M., and Kimura, M. (2001). Neurons in the thalamic CM-Pf complex supply striatal neurons with information about behaviorally significant sensory events. J. Neurophysiol. 85, 960-976.

May, P. J. (2006). The mammalian superior colliculus: laminar structure and connections. Prog. Brain Res. 151, 321-378. doi: 10.1016/s0079-6123(05) 51011-2

McHaffie, J. G., Stanford, T. R., Stein, B. E., Coizet, V., and Redgrave, P. (2005). Subcortical loops through the basal ganglia. Trends Neurosci. 28, 401-407. doi: 10.1016/j.tins.2005.06.006

Mink, J. W. (1996). The basal ganglia: focused selection and inhibition of competing motor programs. Prog. Neurobiol. 50, 381-425. doi: 10.1016/s03010082(96)00042-1

Morris, G., Arkadir, D., Nevet, A., Vaadia, E., and Bergman, H. (2004). Coincident but distinct messages of midbrain dopamine and striatal tonically active neurons. Neuron 43, 133-143. doi: 10.1016/j.neuron.2004.06.012

Parent, A., and Hazrati, L. N. (1995). Functional anatomy of the basal ganglia. I. The cortico-basal ganglia-thalamo-cortical loop. Brain Res. Brain Res. Rev. 20, 91-127. doi: 10.1016/0165-0173(94)00007-c

Parent, A., Mackey, A., and De Bellefeuille, L. (1983). The subcortical afferents to caudate nucleus and putamen in primate: a fluorescence retrograde double labeling study. Neuroscience 10, 1137-1150. doi: 10.1016/0306-4522(83) 90104-5

Parent, M., and Parent, A. (2005). Single-axon tracing and three-dimensional reconstruction of centre median-parafascicular thalamic neurons in primates. J. Comp. Neurol. 481, 127-144. doi: 10.1002/cne.20348

Raju, D. V., Shah, D. J., Wright, T. M., Hall, R. A., and Smith, Y. (2006). Differential synaptology of vGluT2-containing thalamostriatal afferents between the patch and matrix compartments in rats. J. Comp. Neurol. 499, 231-243. doi: 10. 1002/cne.21099

Redgrave, P., Coizet, V., Comoli, E., McHaffie, J. G., Leriche, M., Vautrelle, N., et al. (2010). Interactions between the Midbrain superior colliculus and the Basal Ganglia. Front. Neuroanat. 4:132. doi: 10.3389/fnana.2010.00132

Redgrave, P., Gurney, K., and Reynolds, J. (2008). What is reinforced by phasic dopamine signals? Brain Res. Rev. 58, 322-339. doi: 10.1016/j.brainresrev.2007. 10.007

Redgrave, P., Marrow, L., and Dean, P. (1992). Topographical organization of the nigrotectal projection in rat: evidence for segregated channels. Neuroscience 50 , 571-595. doi: 10.1016/0306-4522(92)90448-b

Redgrave, P., Prescott, T. J., and Gurney, K. (1999). The basal ganglia: a vertebrate solution to the selection problem? Neuroscience 89, 1009-1023. doi: 10 . 1016/s0306-4522(98)00319-4

Redgrave, P., Vautrelle, N., and Reynolds, J. N. J. (2011). Functional properties of the basal ganglia's re-entrant loop architecture: selection and reinforcement. Neuroscience 198, 138-151. doi: 10.1016/j.neuroscience.2011. 07.060

Reiner, A., Jiao, Y., Del Mar, N., Laverghetta, A. V., and Lei, W. L. (2003). Differential morphology of pyramidal tract-type and intratelencephalically projectingtype corticostriatal neurons and their intrastriatal terminals in rats. J. Comp. Neurol. 457, 420-440. doi: 10.1002/cne.10541

Reiner, A., Medina, L., and Veenman, C. L. (1998). Structural and functional evolution of the basal ganglia in vertebrates. Brain Res. Brain Res. Rev. 28, 235285. doi: 10.1016/s0165-0173(98)00016-2

Reynolds, J. N., Hyland, B. I., and Wickens, J. R. (2001). A cellular mechanism of reward-related learning. Nature 413, 67-70. doi: 10.1038/35092560

Reynolds, J. N. J., and Wickens, J. R. (2002). Dopamine-dependent plasticity of corticostriatal synapses. Neural Netw. 15, 507-521. doi: 10.1016/s08936080(02)00045-x

Reynolds, J. N. J., and Wickens, J. R. (2004). The corticostriatal input to giant aspiny interneurons in the rat: a candidate pathway for synchronising the response to reward-related cues. Brain Res. 1011, 115-128. doi: 10.1016/j.brainres.2004.03. 026

Sadikot, A. F., Parent, A., Smith, Y., and Bolam, J. P. (1992). Efferent connections of the centromedian and parafascicular thalamic nuclei in the squirrel monkey: a light and electron microscopic study of the thalamostriatal projection in relation to striatal heterogeneity. J. Comp. Neurol. 320, 228-242. doi: 10.1002/cne. 903200207

Sahibzada, N., Dean, P., and Redgrave, P. (1986). Movements resembling orientation or avoidance elicited by electrical stimulation of the superior colliculus in rats. J. Neurosci. 6, 723-733.

Schultz, W., Dayan, P., and Montague, P. R. (1997). A neural substrate of prediction and reward. Science 275, 1593-1599. doi: 10.1126/science.275.5306.1593

Schulz, J. M., Pitcher, T. L., Savanthrapadian, S., Wickens, J. R., Oswald, M. J., and Reynolds, J. N. J. (2011). Enhanced high-frequency membrane potential fluctuations control spike output in striatal fast-spiking interneurones in vivo. J. Physiol. 589, 4365-4381. doi: 10.1113/jphysiol.2011.212944

Schulz, J. M., and Reynolds, J. N. J. (2013). Pause and rebound: sensory control of cholinergic signaling in the striatum. Trends Neurosci. 36, 41-50. doi: 10.1016/j. tins.2012.09.006

Schulz, J. M., Redgrave, P., Mehring, C., Aertsen, A., Clements, K. M., Wickens, J. R., et al. (2009). Short-latency activation of striatal spiny neurons via subcortical visual pathways. J. Neurosci. 29, 6336-6347. doi: 10.1523/jneurosci.4815-08. 2009

Shen, W., Tian, X., Day, M., Ulrich, S., Tkatch, T., Nathanson, N. M., et al. (2007). Cholinergic modulation of Kir2 channels selectively elevates dendritic excitability in striatopallidal neurons. Nat. Neurosci. 10, 1458-1466. doi: 10. 1038/nn 1972

Sidibé, M., Bevan, M. D., Bolam, J. P., and Smith, Y. (1997). Efferent connections of the internal globus pallidus in the squirrel monkey: I. Topography and synaptic organization of the pallidothalamic projection. J. Comp. Neurol. 382, 323-347. doi: 10.1002/(sici)1096-9861(19970609)382:3<323::aid-cne3>3.0. co;2-5

Sidibé, M., Pare, J.-F., and Smith, Y. (2002). Nigral and pallidal inputs to functionally segregated thalamostriatal neurons in the centromedian/parafascicular intralaminar nuclear complex in monkey. J. Comp. Neurol. 447, 286-299. doi: 10. 1002/cne.10247

Sidibé, M., and Smith, Y. (1996). Differential synaptic innervation of striatofugal neurones projecting to the internal or external segments of the globus pallidus by thalamic afferents in the squirrel monkey. J. Comp. Neurol. 365, 445-465. doi: 10.1002/(sici)1096-9861(19960212)365:3<445::aid-cne8>3.3. $\mathrm{co} ; 2-8$

Sidibé, M., and Smith, Y. (1999). Thalamic inputs to striatal interneurons in monkeys: synaptic organization and co-localization of calcium binding proteins. Neuroscience 89, 1189-1208. doi: 10.1016/s0306-4522(98)00367-4

Smith, Y., and Parent, A. (1986). Differential connections of caudate nucleus and putamen in the squirrel monkey (Saimiri sciureus). Neuroscience 18, 347-371. doi: 10.1016/0306-4522(86)90159-4

Smith, Y., Raju, D. V., Pare, J.-F., and Sidibe, M. (2004). The thalamostriatal system: a highly specific network of the basal ganglia circuitry. Trends Neurosci. 27, 520527. doi: 10.1016/j.tins.2004.07.004

Sparks, D. L. (1986). Translation of sensory signals into commands for control of saccadic eye movements: role of primate superior colliculus. Physiol. Rev. 66, $118-171$.

Stein, B. E., and Gaither, N. S. (1981). Sensory representation in reptilian optic tectum: some comparisons with mammals. J. Comp. Neurol. 202, 69-87. doi: 10. 1002/cne.902020107

Steriade, M., and Glenn, L. L. (1982). Neocortical and caudate projections of intralaminar thalamic neurons and their synaptic excitation from midbrain reticular core. J. Neurophysiol. 48, 352-371.

Suzuki, T., Miura, M., Nishimura, K., and Aosaki, T. (2001). Dopamine-dependent synaptic plasticity in the striatal cholinergic interneurons. J. Neurosci. 21, 64926501.

Takada, M., Itoh, K., Yasui, Y., Sugimoto, T., and Mizuno, N. (1985). Topographical projections from the posterior thalamic regions to the striatum in the cat, with reference to possible tecto-thalamo-striatal connections. Exp. Brain Res. 60, 385396. doi: 10.1007/bf00235934

Takada, M., Tokuno, H., Ikai, Y., and Mizuno, N. (1994). Direct projections from the entopeduncular nucleus to the lower brainstem in the rat. J. Comp. Neurol. 342, 409-429. doi: 10.1002/cne.903420308 
Tsumori, T., Yokota, S., Ono, K., and Yasui, Y. (2003). Nigrothalamostriatal and nigrothalamocortical pathways via the ventrolateral parafascicular nucleus. Neuroreport 14, 81-86. doi: 10.1097/00001756-20030120000016

Van der Werf, Y. D., Witter, M. P., and Groenewegen, H. J. (2002). The intralaminar and midline nuclei of the thalamus. Anatomical and functional evidence for participation in processes of arousal and awareness. Brain Res. Brain Res. Rev. 39, 107-140. doi: 10.1016/s0165-0173(02) 00181-9

Westby, G. W., Keay, K. A., Redgrave, P., Dean, P., and Bannister, M. (1990). Output pathways from the rat superior colliculus mediating approach and avoidance have different sensory properties. Exp. Brain Res. 81, 626-638. doi: 10 1007/bf02423513

Wichmann, T., and DeLong, M. R. (1996). Functional and pathophysiological models of the basal ganglia. Curr. Opin. Neurobiol. 6, 751-758. doi: 10.1016/s09594388(96)80024-9

Wickens, J. R., Reynolds, J. N. J., and Hyland, B. I. (2003). Neural mechanisms of reward-related motor learning. Curr. Opin. Neurobiol. 13, 685-690. doi: 10. 1016/j.conb.2003.10.013
Wurtz, R. H., and Albano, J. E. (1980). Visual-motor function of the primate superior colliculus. Annu. Rev. Neurosci. 3, 189-226. doi: 10.1146/annurev.ne. 03.030180 .001201

Conflict of Interest Statement: The authors declare that the research was conducted in the absence of any commercial or financial relationships that could be construed as a potential conflict of interest.

Received: 30 January 2014; accepted: 19 March 2014; published online: 02 April 2014. Citation: Fisher SD and Reynolds JNJ (2014) The intralaminar thalamus - an expressway linking visual stimuli to circuits determining agency and action selection. Front. Behav. Neurosci. 8:115. doi: 10.3389/fnbeh.2014.00115

This article was submitted to the journal Frontiers in Behavioral Neuroscience.

Copyright $\odot 2014$ Fisher and Reynolds. This is an open-access article distributed under the terms of the Creative Commons Attribution License (CC BY). The use, distribution or reproduction in other forums is permitted, provided the original author(s) or licensor are credited and that the original publication in this journal is cited, in accordance with accepted academic practice. No use, distribution or reproduction is permitted which does not comply with these terms. 